Table 1 - Groups' characteristics and scales descriptives

\section{Quality of life and childhood epilepsy}

\section{Qualidade de vida e epillepsia da infância}

Dear Editor,

Among severe neurological conditions, epilepsy is the most prevalent, occurring in $0.5 \%$ of the general population, with a higher prevalence among children and adolescents. Several studies point that chronic diseases produce higher susceptibility to psychiatric morbidity when compared to control groups. The World Health Organization developed an instrument to assess the quality of life in the framework of a collaborative multicentre project (WHOQOL Group), which has been widely applied in Brazil in its Portuguese version. ${ }^{1}$ The Quality of life (QOL) approach has been considered one of the best alternatives for the psychic evaluation of children with chronic conditions. Unfortunately, we still cannot describe such development of instruments concerning the evaluation of the quality of life evaluation among children and adolescents. We present a study of QOL in children and adolescents with epilepsy (CAWE) using a self-applying questionnaire and comparing its performance to questionnaires applied to parents.

We evaluated 28 outpatients with childhood epilepsy diagnosis and follow-up, aged 4 to 12 years and 11 months, and 28 matched healthy schoolchildren, and we also interviewed the parents of both groups. The Autoquestionnaire Qualité de Vie Enfant Imagé (AUQEI), validated for the local population, ${ }^{2}$ was applied to children and adolescents. We also applied the Children's Global Assessment Scale $(\mathrm{CGAS})^{3}$ and the Vineland Adaptive Behavior Scale (VINELAND), ${ }^{4}$ the

\begin{tabular}{|c|c|c|}
\hline & Epileptic & Control \\
\hline n (\%) & $28(100)$ & $28(100)$ \\
\hline \multicolumn{3}{|l|}{ (T test) } \\
\hline \multicolumn{3}{|l|}{ Sex } \\
\hline Male (5) & $14(50)$ & $12(42.86)$ \\
\hline Female (\%) & $14(50)$ & $16(57.14)$ \\
\hline \multicolumn{3}{|l|}{ Age (years) } \\
\hline$<8(\%)$ & $11(39.29)$ & $16(57.14)$ \\
\hline $\begin{array}{l}8-12(\%) \\
\left(t_{\circ} 1.83\right)\end{array}$ & $17(60.71)$ & $12(42.86)$ \\
\hline $\begin{array}{l}\text { CGAS Score: } \mathbf{m}(\mathbf{s d}) \\
\left(t_{\circ} 1.35\right)\end{array}$ & $86.32(17.35)$ & $91.11(6.21)$ \\
\hline $\begin{array}{l}\text { Total Vineland Score: } \mathbf{m}(\mathbf{s d}) \\
\left(\mathrm{t}_{\circ} 6.28\right)\end{array}$ & $69.71(17.76)$ & $94.75(10.64)$ \\
\hline $\begin{array}{l}\text { Communication Vineland Score: } \mathbf{m} \text { (sd) } \\
(t, 5.15)\end{array}$ & 75.64 (18.89) & $100.79(16.97)$ \\
\hline $\begin{array}{l}\text { Daily Activities Vineland Score: } \mathbf{m} \text { (sd) } \\
(\mathrm{t}, 4.79)\end{array}$ & $72.39(24.31)$ & $94.79(8.92)$ \\
\hline $\begin{array}{l}\text { Social Skills Vineland Score: } \mathbf{m}(\mathbf{s d}) \\
\left(t_{\circ} 5.08\right)\end{array}$ & $74.89(13.80)$ & $93.61(13.28)$ \\
\hline $\begin{array}{l}\text { AUQEI Score: } \mathbf{m}(\mathbf{s d}) \\
\left(t_{\circ} 2.99\right)\end{array}$ & $53.36(5.72)$ & $58.43(6.69)$ \\
\hline
\end{tabular}

n: number of cases; CGAS: Children's Global Assessment Scale; m: mean; sd: standard deviation; AUQEI: Autoquestionnaire Qualité de Vie Enfant Imagé

$T$ test: $T$ independent test, $<0.05$

last one composed by a total score and three subordinate domains: communication, daily living skills and socialization. CGAS and VINELAND were applied to parents. T independent test was used to analyze the data. The significance level adopted was of $5 \%$.

Results are presented in Table 1. No significant differences were observed between age groups ( $\left.t_{0} 1.83\right)$ and as to CGAS scores ( $t_{0}$ 1.35). Significant differences were observed as to Vineland total scores. CAWE had worse performance than controls ( $t$ 6.28), as well as regarding to communication ( $\left.t_{0} 5.15\right)$, daily living skills (t 4.79) and socialization (t 5.08) domains results. Significant differences were observed for AUQEI results ( $t_{0}$ 2.99). The CAWE group had worse results than matched controls.

The majority of studies investigating the impact of epilepsy on QOL of children and adolescents have used proxy opinions, with clinician and/or parent reports. This study highlights the need for research investigating QOL from the direct perspective of children and considering issues in the context of a developmental perspective. For this purpose, in this study we used a self-reporting questionnaire. CAWE clearly demonstrated inferior performance in regard to self perception of QOL and to the development of an adaptive behavior, even though these went unrecognized by their parents, as shown by CGAS. 
leukemia and juvenile rheumatoid arthritis, performed worse in adaptive behavior development. ${ }^{5}$ In this context, CAWE are no exception. With regardto global functioning, obtained from CGAS, there were no significant statistical differences in our study, demonstrating the absence of a higher incidence of complaints of this nature arising from parents, in relation to their chronic disease.

Adequate instruments should be necessarily developed to assess perceptions of disease and treatment from chronic pediatric patients themselves, aiming to increase attention given to this growing and needing population in terms of mental health. ${ }^{5}$

Evelyn Kuczynski

Grupo de Atendimento em Psiquiatria Infantil ao Epiléptico (GAPIE), Instituto de Psiquiatria, Hospital das Clínicas,

Faculdade de Medicina, Universidade de São Paulo (USP), São Paulo (SP), Brazil

Projeto Distúrbios do Desenvolvimento, Departamento de Psicologia Clínica, Instituto de Psicologia, Universidade de São Paulo (USP),

São Paulo (SP), Brazil
Maria Sigride Thomé-Souza

Grupo de Atendimento em Psiquiatria Infantil ao Epiléptico (GAPIE), Instituto de Psiquiatria, Hospital das Clínicas, Faculdade de Medicina, Universidade de São Paulo (USP), São Paulo (SP), Brazil Laboratório de Neurofisiologia Clínica, Instituto de Psiquiatria, Hospital das Clínicas, Faculdade de Medicina, Universidade de São Paulo (USP), São Paulo (SP), Brazil

Lia A Fiore, Kette D R Valente Laboratório de Neurofisiologia Clínica, Instituto de Psiquiatria, Hospital das Clínicas, Faculdade de Medicina, Universidade de São Paulo (USP), São Paulo (SP), Brazil

Francisco B Assumpção Jr. Projeto Distúrbios do Desenvolvimento, Departamento de Psicologia Clínica, Instituto de Psicologia, Universidade de São Paulo (USP), São Paulo (SP), Brazil

\section{Disclosures}

\begin{tabular}{|c|c|c|c|c|c|c|c|}
\hline $\begin{array}{c}\text { Writting group } \\
\text { member }\end{array}$ & Employment & $\begin{array}{l}\text { Research } \\
\text { grant }^{1}\end{array}$ & $\begin{array}{l}\text { Other research grant or } \\
\text { medical continuous education }\end{array}$ & $\begin{array}{l}\text { Spekear's } \\
\text { honoraria }\end{array}$ & $\begin{array}{c}\text { Ownership } \\
\text { interest }\end{array}$ & $\begin{array}{c}\text { Consultant/ } \\
\text { Advisory board }\end{array}$ & Other $^{3}$ \\
\hline Evelyn Kuczynski & USP & -- & - & - & -- & - & - \\
\hline $\begin{array}{l}\text { Maria Sigride } \\
\text { Thomé-Souza }\end{array}$ & USP & - & - & - & - & - & - \\
\hline Lia A Fiore & USP & -- & - & - & - & - & - \\
\hline $\begin{array}{l}\text { Kette D. R. } \\
\text { Valente }\end{array}$ & USP & - & - & - & - & - & - \\
\hline $\begin{array}{l}\text { Francisco B. } \\
\text { Assumpçāo Jr. }\end{array}$ & USP & - & - & - & - & - & - \\
\hline
\end{tabular}

* Modest

** Significant

** Significant. Amounts given to the author's institution or to a colleague for research in which the author has participation, not directly to the author. Note: USP = Universidade de São Paulo.

For more information, see instructions for authors.

\section{References}

1. Fleck MPA e colaboradores. A avaliação de qualidade de vida: guia para profissionais da saúde. Porto Alegre: Artmed; 2008.

2. Assumpção Jr FB, Kuczynski E, Sprovieri MHS, Aranha EMG. Escala de avaliação de qualidade de vida (AUQEI - Autoquestionnaire qualité de vie enfant imagé): validade e confiabilidade de uma escala para qualidade de vida em crianças de 4 a 12 anos. Arq Neuropsiquiatr. 2000;58(1):119-27.

3. Shaffer D, Gould MS, Brasic J, Ambrosini P, Fisher P, Bird H, Aluwahlia S. A children's global assessment scale (CGAS). Arch Gen Psychiatr. 1983;40(11):1228-31.

4. Sparrow SS, Balla DA, Cicchetti DV. Vineland adaptive behavior scales. Circle Pines (MN): American Guidance Service; 1984.

5. Kuczynski E, Silva CAA, Cristófani LM, Kiss MHB, Odone Filho V, Assumpção Jr FB. Evaluación de la calidad de vida en niños y adolescentes portadores de enfermedades crónicas y/o incapacitadoras: un estudio brasileño. An Pediatr. 2003;58(6):550-5. 